\title{
Evaluation of Acoustic and Vibratory Characteristics of Impact Noise Due to Rail Joints
}

\author{
Takeshi SUEKI \\ Toshiki KITAGAWA \\ Tsugutoshi KAWAGUCHI \\ Noise Analysis Laboratory, Environmental Engineering Division \\ Impact noise induced by rail joints is one of the major sources of wayside noise on rail- \\ ways. Field tests were conducted around a rail joint on a commercial meter-gauged railway \\ line. It was found out that the sound power of the total impact noise increased proportionally \\ to train speed to the power of 2.4 in the speed range of $40 \mathrm{~km} / \mathrm{h}$ to $100 \mathrm{~km} / \mathrm{h}$. Estimation of \\ contribution to impact noise from rails and sleepers based on their vibration showed that \\ noise generated by sleepers is the dominant source of impact noise below $500 \mathrm{~Hz}$, whilst above \\ $1 \mathrm{kHz}$ it is from the rails. A prediction model indicated that modifying track parameters \\ could potentially reduce impact noise.
}

Keywords: railway noise, impact noise, rail joint, rail vibration, sleeper vibration, track parameter

\section{Introduction}

When trains pass over rail discontinuities such as joints, strong impulsive noises, hereinafter referred to as "impact noise", are produced in addition to the normal rolling noise generated along continuous rails. This impact noise can be reduced by installing continuous welded rails. However, continuous welded rails cannot be used at turnouts and on steel bridges, or when current insulation is required for signaling control.

In order to investigate the characteristics of impact noise, Kitagawa et al. measured track and wheel vibrations at low speeds (up to $35 \mathrm{~km} / \mathrm{h}$ ) along the test track at the Railway Technical Research Institute [1]. Their measurements showed that i) the acoustic power of the impact noise is proportional to the $2 \mathrm{nd}-3 \mathrm{rd}$ power of train velocity; and ii) when one of the two facing rails at a rail joint is excited by an impulse hammer, the frequency responses of the struck rail and its counterpart differ above $800 \mathrm{~Hz}$.

To theoretically investigate the vibratory behavior of the rail joints, Kitagawa et al. then modeled the rail as a pair of semi-finite Timoshenko beams connected by a complex spring [1]. The noise predictions of this model favorably agree with those measured in the running test. However, this model was verified only in the speed range up to $35 \mathrm{~km} / \mathrm{h}$.

In order to investigate the vibratory and acoustic characteristics of the impact noise caused by the train running at a higher speed than in previous studies, noise and vibration were measured at a rail joint on a commercial metergauged railway line for train speeds ranging from $40 \mathrm{~km} /$ $\mathrm{h}$ to $100 \mathrm{~km} / \mathrm{h}$. This paper presents the results of both the experiments conducted during train passage and of the stationary experiments using impact hammer excitation. This paper also gives estimations and presents the contribution of track components to total impact noise. Furthermore, attempts were made to verify validity of the prediction model by comparing impact noise estimated with the model and that measured at the rail joint, and to estimate the influence of track parameters on impact noise.

\section{Outline of tracks for measurement}

Measurements were taken at one joint and along one continuous rail section, as shown in Fig. 1. These tracks were composed of ballast and $60 \mathrm{~kg}$ rails. The static stiffness of the rail pads was $60 \mathrm{MN} / \mathrm{m}$. The joint and continuous rail sections are supported on wooden and concrete monoblock sleepers. A 'supported joint' system was used, which means that a dedicated sleeper was providing support directly below the joint.

Figure 1 also shows the position of the sensors, i.e. accelerometers and microphones. The sensors were also used to measure the frequency response in the hammer excitation tests described in chapter three, and the time series signals during trains passage described in the chapter four.

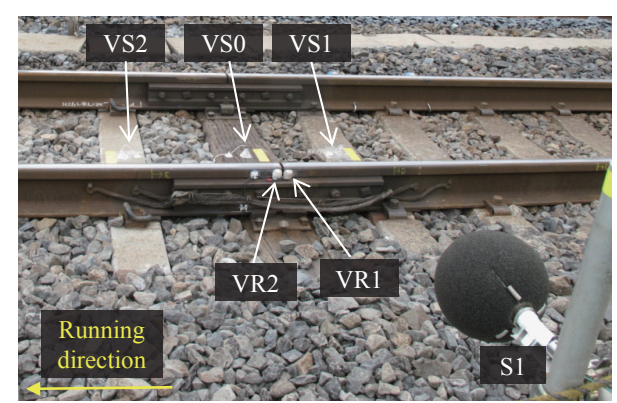

(a) Joint section

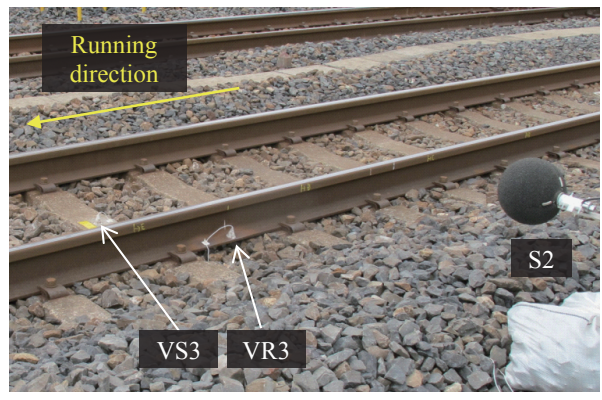

(b) Continuous rail section

Fig. 1 Tracks used for the measurements 
Details of the measurements made using the sensors are given in the relevant chapters below.

Figure 2 indicates the joint geometry measured with a rail roughness measuring instrument, HTR-8A manufactured by Harada seisakusho Co. Ltd. This instrument can be used to measure roughness with a precision of $10^{-6}$ $\mathrm{m}$ at intervals of $10^{-3} \mathrm{~m}$ in the train's travelling direction. Dimensions were also measured directly using a ruler. The joint section had a gap of $8.55 \mathrm{~mm}$ in length and a stepdown height of $0.067 \mathrm{~mm}$.

\section{Track vibratory characteristics obtained in ham- mer excitation tests}

\subsection{Measurement process}

To investigate the relationship between track vibratory characteristics and impact noise, excitation tests were conducted using the impulse hammer, Dytran models 5800B4 (up to $10 \mathrm{kHz}$ ), as shown in Fig. 3. Accelerometers were installed on the tracks as shown in Fig. 1. The accelerometers used were Dytran 3033B2 (at sites VR1 and VR2), and 3056B1 and 3056B2 (at other sites). A force was exerted at a point close to the accelerometer and its frequency response function (point accelerance) was estimated using FFT analysis.

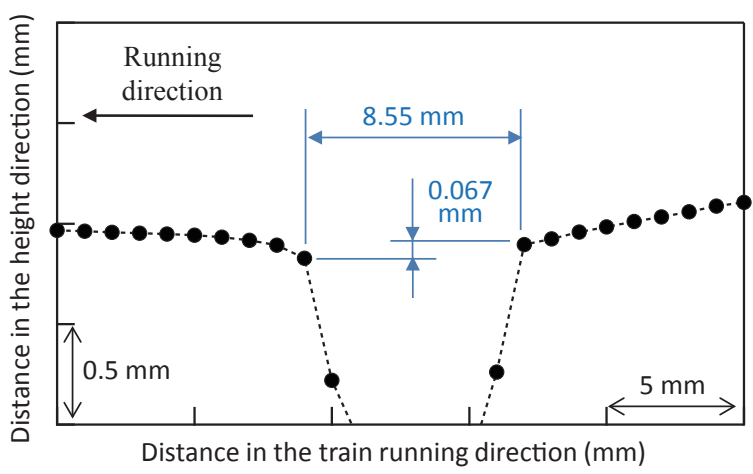

Fig. 2 Dimensions of the joint

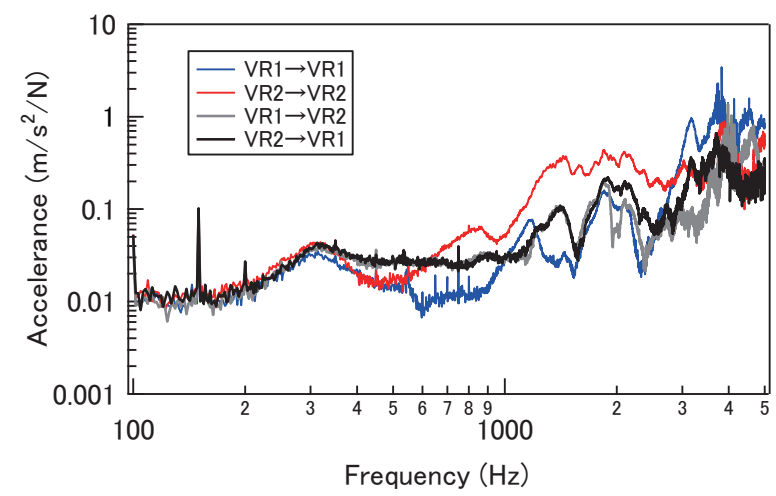

(a) Rail responses

\subsection{Frequency response at the joint section}

Figure 4 shows frequency responses measured in the impact hammer excitation test. In the figure, ' $\mathrm{X} \rightarrow \mathrm{Y}$ ' means 'frequency response at position $\mathrm{Y}$ by exciting the rail at position $X$ '. The figure only shows results in the frequency range where a sufficient signal-to-noise ratio in measurements was obtained. The peaks at $100 \mathrm{~Hz}, 150 \mathrm{~Hz}$ and $200 \mathrm{~Hz}$ in Fig. 4 were due to overlapping of power supply noise.

Figure 4 (a) shows the frequency responses of one of the rails at the joint when the other is excited. In Fig. 4 (a) the point accelerances 'VR1 $\rightarrow \mathrm{VR} 1$ ' and 'VR2 $\rightarrow \mathrm{VR} 2$ ' are similar in the range below the $400 \mathrm{~Hz}$ band. This indicates that the two rails vibrate together in the frequency range below the $400 \mathrm{~Hz}$ band with consideration of the phase results (the phase results are not shown here). Above the 400 $\mathrm{Hz}$ band, the rails vibrate independently despite both rails being tightly fastened with one fishplate. The results of 'VR1 $\rightarrow$ VR2' and 'VR2 $\rightarrow$ VR1' are almost the same below 3 $\mathrm{kHz}$. This suggests that the vibration transmitted through the fishplate does not depend on the vibration at the excitation point.

Figure 4 (b) shows the transferred accelerance between the rail and the sleepers. In the joint sections, the results from excitation of VR2 alone are shown, since the impact force when a train passes excites only one side where the

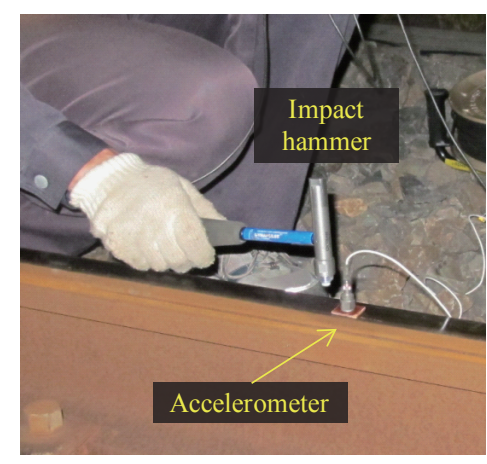

Fig. 3 Impact hammer excitaiton

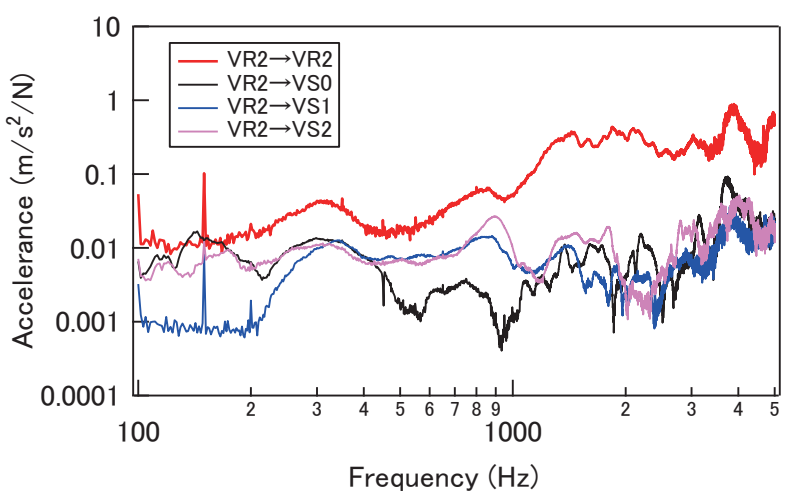

(b) Sleeper responses

Fig. 4 Results of impact excitation when rail is excited 
joint is. This shows that the characteristics of the respective sleeper vibrations are different although they are laid in the same joint section.

\subsection{Difference in frequency response between joint and continuous rail section}

Figure 5 shows the point accelerance at three points: VR1 (the joint section), a point $1.8 \mathrm{~m}$ away from VR1, and VR3 (continuous rail section).

The results for VR1 clearly differ from those of VR3, and the point $1.8 \mathrm{~m}$ away from VR1. This indicates that the vibratory characteristics of the rail section with a joint are different from those of the continuous rail section. Similar characteristics were observed from the VR3 results and those from the point $1.8 \mathrm{~m}$ away from VR1. This suggests that the point accelerance at the point about $1.8 \mathrm{~m}$ away from the joint is similar to that of the continuous rail section. These results, that the accelerance of the track only a few meters away from the joint has similar characteristics to that of the continuous rail section, indicate that the vibratory characteristics of the track near the joint is locally different.

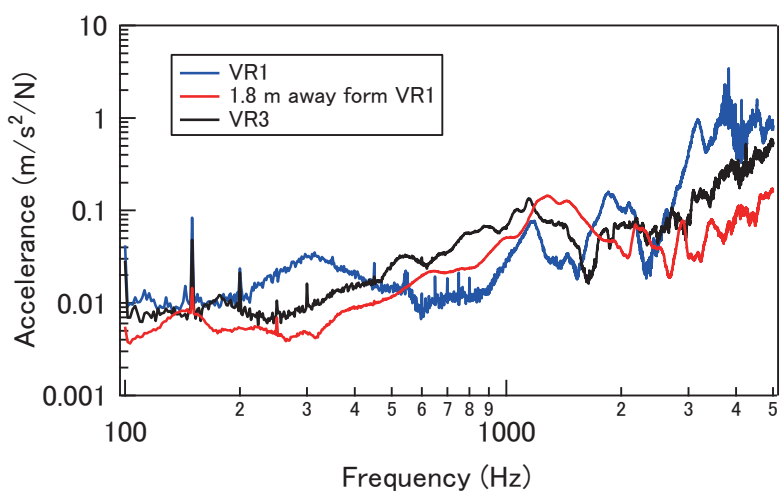

Fig. 5 Comparison of frequency responses at their excitation points

\section{Noise and vibratory characteristics of the joint section during train passage}

\subsection{Measurement during the passage of a train}

Using accelerometers and microphones, the noise and vibrations produced by a passing train were measured along the joint and continuous rail sections, as shown in Fig. 1. The same accelerometers as those described in the chapter three were used. The microphones used were RION UC-30. Estimations of the train velocity of each bogie were made from the accelerations at VR1 and VR2.

Sound pressure was converted into decibels by frequency weighting 'A' and time weighting 'FAST', respectively. Acceleration was also first converted into vibration velocity and then into decibels using the same weightings as for sound pressure. Figure 6 shows typical time histories of Aweighted sound pressure levels at S1 and S2.

Data was arranged by averaged power around the peaks corresponding to the passage of individual wheel sets (see Fig. 6). Due to the time weighting, the impact noise and related vibration generated by the fore wheel sets affected the results of the aft wheel sets. The third set of wheels on each car had the smallest effect on the other sets of wheels; consequently the analysis focused only on the data from the third wheel set on each train car (Fig. 6).

\subsection{Dependence of impact noise on velocity}

The overall A-weighted sound pressure measured at $\mathrm{S} 1$ and S2 are plotted against train velocity in Fig. 7. To investigate the influence of car weight and motor fan noise, these results were classified by type of car, namely motor car or trailer car. ' $M$ ' or ' $T$ ' in the legend of the figure mean motor car or trailed car, respectively. The lines in the figure are regression lines estimated from the measured data.

As the velocity increases from $40 \mathrm{~km} / \mathrm{h}$ to $100 \mathrm{~km} / \mathrm{h}$, the impact noise at $\mathrm{S} 1$ increases with the $24 \log _{10} V$, where $V$ is the train velocity. Compared with the continuous rail section results, the impact noise in the joint section is 5-8 $\mathrm{dB}$ greater. The measured data in the continuous rail sec-

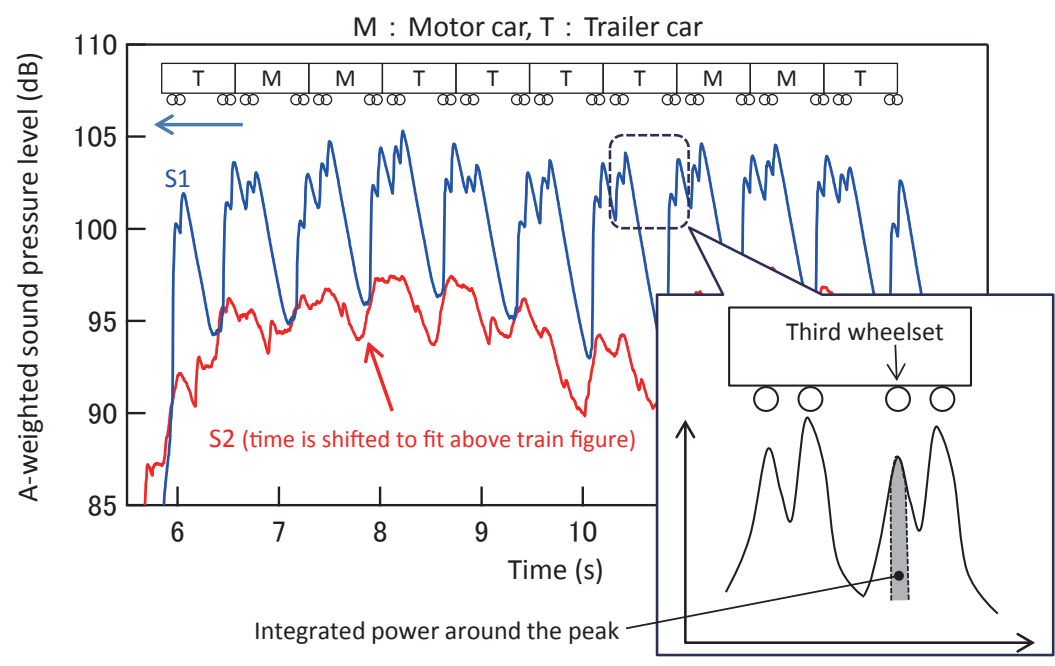

Fig. 6 An example of sound pressure level against time 
tion include the influence of the joint which is around 10 $\mathrm{m}$ away from the continuous section. Therefore, the level differences of sound pressure between the joint and continuous rail sections may be greater than those mentioned above.

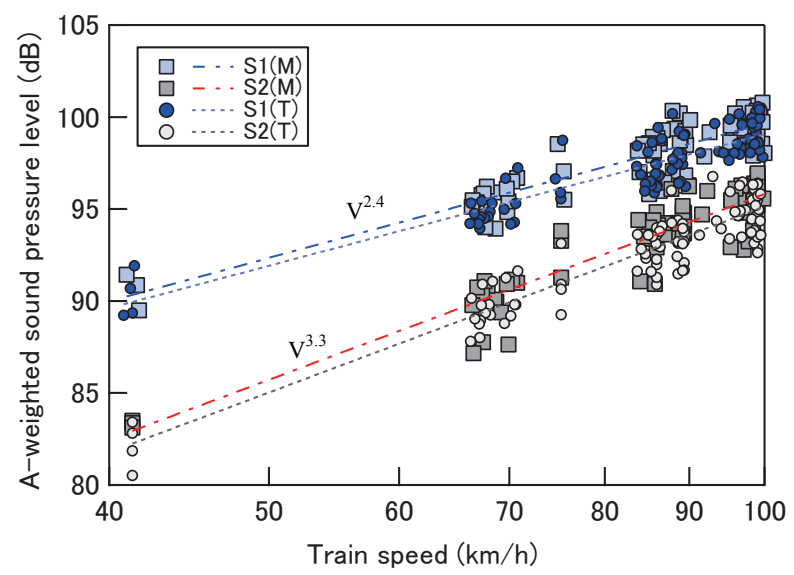

Fig. 7 Overall sound pressure levels as functions of train speed

((M) and ( $\mathrm{T})$ indicates motor and trailer car respectively.)
Figure 7 also shows that the impact noise does not depend on type of car. The weight of a motor car is about $28,000 \mathrm{~kg}$ and that of a trailer car is about $22,000 \mathrm{~kg}$. This difference in weight does not affect the impact noise and the motor fan noise has little effect on the measured data.

\subsection{Frequency characteristics of noise and vibration}

Figure 8 (a) shows the averaged frequency spectra of the impact noise in the $1 / 3$ octave bands at $99 \mathrm{~km} / \mathrm{h}$. The sound pressure level at $\mathrm{S} 1$ is greater than that at $\mathrm{S} 2$ in almost all the frequency bands. In particular, the difference in levels in the 315 and $500 \mathrm{~Hz}$ bands is significantly large. As mentioned below, this is mainly attributable to sleeper noise. Little difference can be seen either between types of car in the frequency spectra.

Figures 8 (b) and (c) show the rail and sleeper vibration spectra. Only the trailer cars results are plotted in the figures. The vibrations below the $500 \mathrm{~Hz}$ band in the joint sections are much greater than in the continuous rail section. This makes the impact noise increase below the 500 $\mathrm{Hz}$ band. Vibrations of the different sleepers in the joint section differ; this is attributed to the vibratory characteristics of sleepers as described in section 3.2.

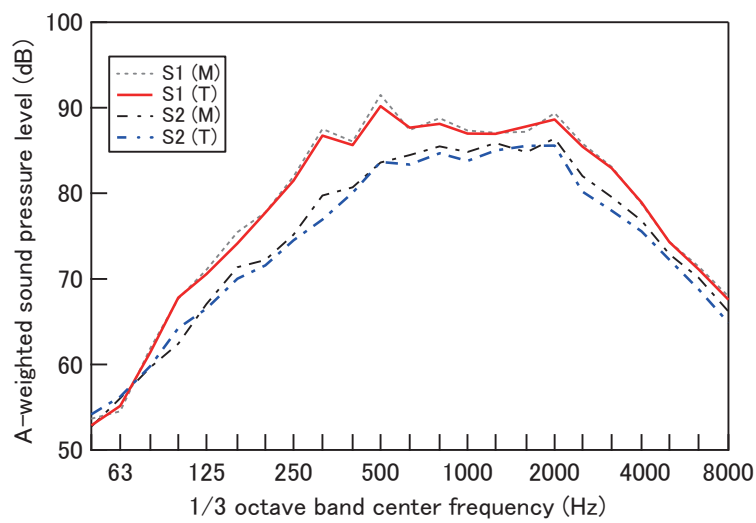

(a) Sound pressure

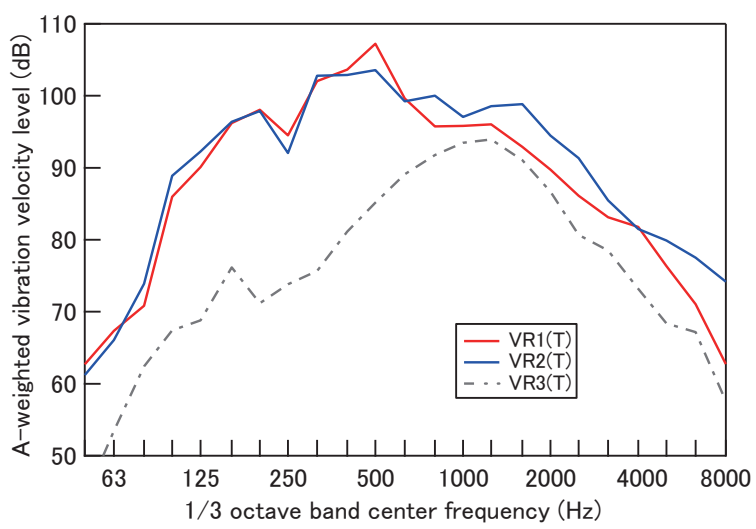

(b) Rail vibration, ref $=5 \times 10^{-8} \mathrm{~m} / \mathrm{s}$

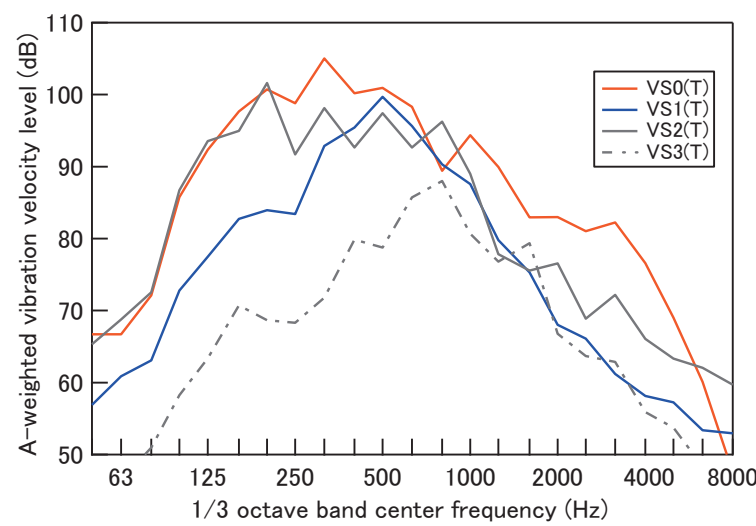

(c) Sleeper vibration, ref $=5 \times 10^{-8} \mathrm{~m} / \mathrm{s}$

Fig. 8 Frequency spectrum over $1 / 3$ octave bands at $99 \mathrm{~km} / \mathrm{h}$ ((M) and $(\mathrm{T})$ indicate motor and trailer car, respectively; rail and sleeper vibrations are shown for the trailer car only.) 


\section{Contribution of different noise sources to impact noise}

\subsection{Estimation of contributions}

In order to develop impact noise reduction measures, it is important to estimate the contribution of different noise sources, such as rails, wheels and sleepers, to the total impact noise. The contribution of these different noise sources to impact noise was estimated using an acoustic model constructed on the basis of vibration results from stationary and running tests. However, since these tests could not measure wheel vibrations, the contribution to noise of the wheels was estimated by subtracting contributions from rail and sleeper noise from the total measured noise. Estimations were made on the assumption that the vehicle was a trailer car in order to ensure no other noise sources were involved, such as motor fan noise.

In the case of the rail, it was assumed that a line source consists of plural point sources on the rail in the half space. Then, at a given measurement point, the sound pressure level generated from the rail, $L_{p r}$, is given by (1).

$$
\begin{array}{r}
L_{p r}=10 \log _{10}\left(\frac{\rho c_{0} \Omega_{r} P\left|v_{r 0}\right|^{2}}{2 \pi} \int_{0}^{\infty} \frac{\mathrm{e}^{-2 \beta x}}{x^{2}+y^{2}} d x\right) \\
-10 \log _{10}\left(W_{r e f}\right)
\end{array}
$$

where $y$ is the distance from the rail to the measurement point; $\rho$, the air density; $c_{0}$, the speed of sound in air; $\Omega_{r}$, the acoustic radiation efficiency of the rail; $P$, the surface area per meter related to the vertical vibration of the rail; $v_{r 0}$, the rail vibration velocity at the reference point; $W_{\text {ref }}$ the reference acoustic power (equal to $1 \times 10^{-12} \mathrm{~W}$ ); $\beta$, the value related to the decay rate of vibrations in the longitudinal direction of the rail; and $x$, the displacement in the longitudinal direction of the rail.

In the case of the sleepers, the noise sources were assumed to be point sources on the respective sleepers in the half space. Then, at a given measurement point, the sound pressure level generated from one sleeper, $L_{p s}$, is given by (2).

$$
L_{p s}=10 \log _{10}\left(\frac{\rho c_{0} v_{s}^{2} S \Omega_{s}}{2 \pi r^{2}}\right)-10 \log _{10}\left(W_{r e f}\right)
$$

where $r$ is the distance from the sleeper to the measurement point; $v_{s}$, the averaged vibration velocity of the vibrating surface; $S$, the area of the vibrating surface; and $\Omega_{s}$, the acoustic radiation efficiency of the sleeper.

The following assumptions are also applied to the estimation of the respective contributions:

- The measured data at VR2 represents rail vibration $v_{r 0}$.

- The noise source of the sleepers is located at the quarterlength of the sleeper from its edge, and half of the top surface of the sleeper vibrates. The sleeper just below the joint and the neighboring sleepers are considered in the estimation of sleeper contribution to noise.

- The vibration decay rate of the continuous rail section is used as that of the joint sections.

- The acoustic radiation efficiency ratio refers to the data given in [2].
- The source is an omnidirectional one.

\subsection{Results obtained through estimation}

The estimated results are shown in Fig. 9. Sleepers are major source of noise below the $500 \mathrm{~Hz}$ band, while sleepers vibrating with a larger amplitude in the joint section contribute even more to impact noise. As mentioned in section 4.3 , the sound pressure in joint sections far exceeds that of continuous sections below the $500 \mathrm{~Hz}$ band. Therefore, it is inferred that the contribution to this increase is mainly due to sleeper vibration.

Above $1 \mathrm{kHz}$, rails become a major source of noise. Wheels appear to significantly contribute to impact noise in the $0.4-1.6 \mathrm{kHz}$ bands and above the $2 \mathrm{kHz}$ band. However, according to the previous studies, wheel contribution to noise was only notable above $2 \mathrm{kHz}$ [1]. Impact noise in the $0.4-1.6 \mathrm{kHz}$ bands is therefore from rail and sleepers. The difference in the $0.4-1.6 \mathrm{kHz}$ bands may be attributable rail vibrations from VR1 being excluded in earlier estimations.

The main components of the total impact noise are around the $0.25-2 \mathrm{kHz}$ bands in Fig. 9. Impact noise is dominated by noise radiated from the track.

\section{Evaluation of the impact noise using the predic- tion model and influence of track parameters}

\subsection{Impact noise prediction model}

This section presents an attempt to apply the prediction model presented in [1] to the joint section, described above. The influences of track parameters on impact noise are also investigated.

Figure 10 shows the impact noise prediction model, which is composed of a wheel model and a track model. The wheel model consists of a mass, a spring and a damper. The track model consists of two semi-finite Timoshenko beams, and masses and springs corresponding to rails, sleepers, rail pads and ballast. To simulate the transmission of vibration through a fishplate, two rails were connected with a complex spring.

In a study on rolling noise, the force between the wheel and rail is described as the force given by the linear contact

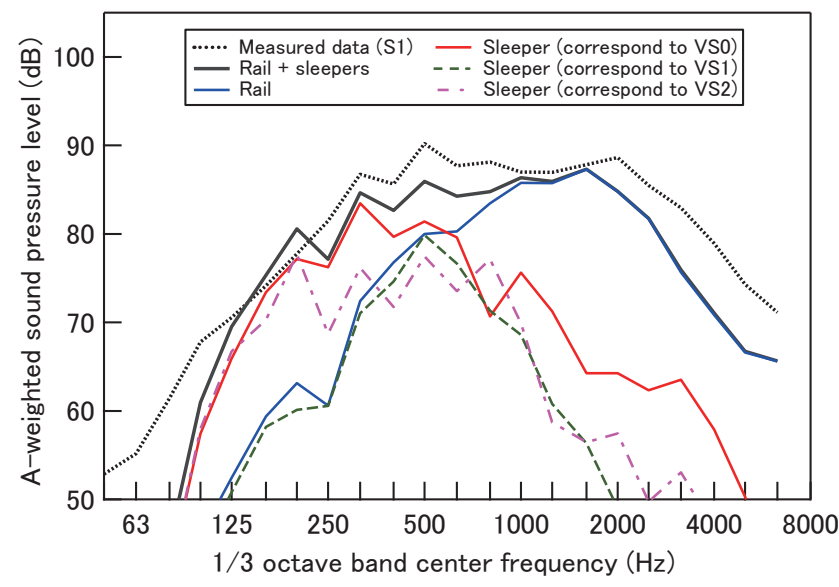

Fig. 9 Estimated contributions of different noise 


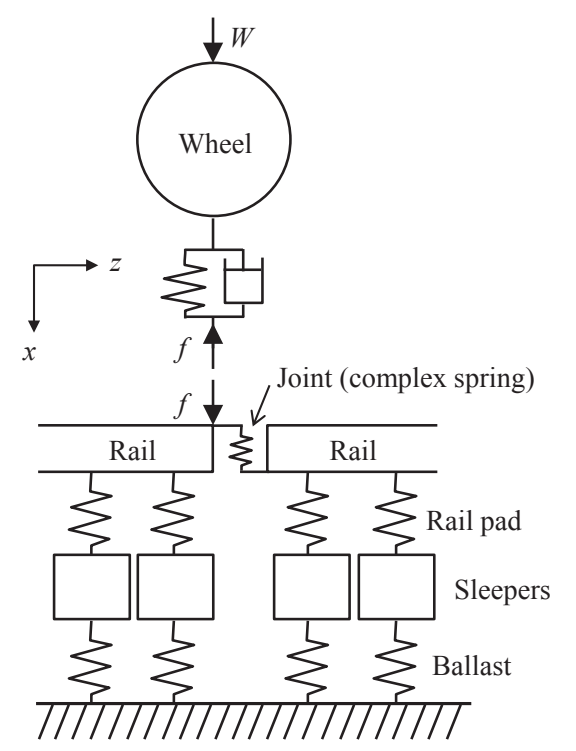

Fig. 10 Impact noise prediction model

spring [3]. The linear contact spring assumes the roughness of the contact surfaces in the order of several microns. However, roughness related to the impact noise can exceed the roughness in the order of microns. In this paper, the contact force is then assumed to be given by (3), which uses a Hertzian contact spring with nonlinear properties [4],

$$
f= \begin{cases}C_{H}\left(x_{w}-x_{r}-r\right)^{\frac{3}{2}} & x_{w}-x_{r}-r>0 \\ 0 & x_{w}-x_{r}-r \leq 0\end{cases}
$$

where $C_{H}$ is the Hertzian constant, $x_{w}, x_{r}$ and $r$ are displacements of wheel, rail and roughness respectively.

The force and vibrations of a rail and a wheel are calculated by the Runge-Kutta method in the time domain. Then, after calculating the equivalent roughness that is equal to the roughness inducing the same exciting force calculated by the prediction model, noise from wheel, rail and sleeper are evaluated using the TWINS model [3].

Figure 11 (a) shows the estimated results from this prediction procedure. The predicted sound pressure level does not completely coincide with the measured level. However, the principal feature of the predicted impact noise agrees with that of the measured noise as indicated by the fact that the predicted contributions from the respective noise sources are similar to the estimation results described in the chapter five.

\subsection{The influence of track parameters on the impact noise}

Modification of track parameters and its purpose are described blow. The modification of the parameters can be achieved from the view point of materials.

(i) Sleeper mass (1.5 times heavier): suppressing sleeper vibration, changing resonance frequency due to sleeper mass and ballast stiffness.

(ii) Rail loss factor (twice greater): Reduction of propagated vibration along the rail.

(iii) Rail pad stiffness (softer by three quarters): Mitigation of excitation force and changing transfer characteris-

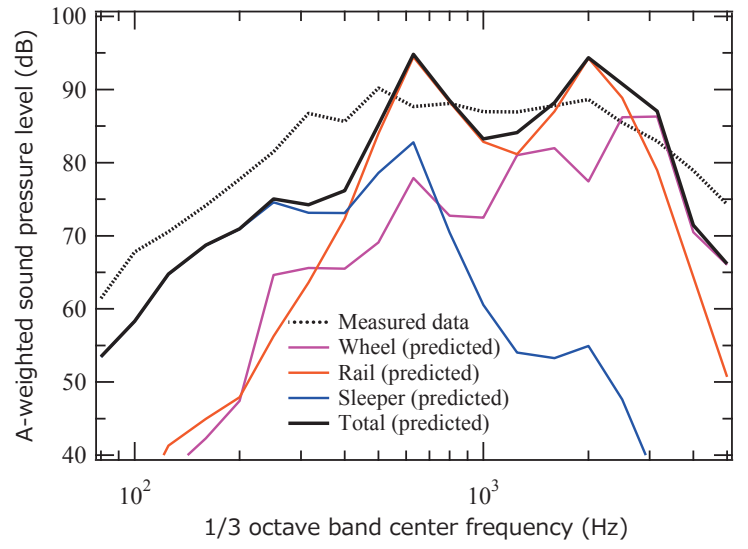

(a) Comparison between the estimated and the measured results

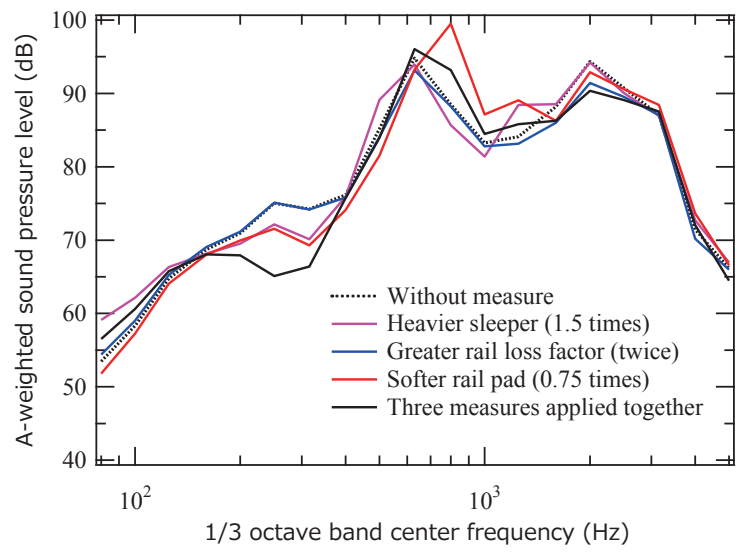

(b) Influence of the track parameters

\section{Fig. 11 Evaluation using the prediction model}

tics from rail to sleepers by modifying the resonance frequency due to the rail mass and rail pad stiffness.

Figure $11(b)$ shows estimated results of the influence of track parameters.

In the case of a heavier sleeper, the sound pressure level around the $250 \mathrm{~Hz}$ band decreased. This is due to the fact that sleeper vibration was reduced by the heavier mass and the vibration from the rail to the sleeper was insulated by the lower resonance frequency attributed to sleeper mass and ballast stiffness. A greater rail loss factor reduces noise in the frequency range between the $1 \mathrm{kHz}$ band and the $3.15 \mathrm{kHz}$ band. This is due to the suppression effect of vibrations propagating by a greater loss factor. Softer rail pads reduce noise around the $250 \mathrm{~Hz}$ band, whilst the sound pressure level above the $800 \mathrm{~Hz}$ band increased. Around the $250 \mathrm{~Hz}$ band, the excitation force was reduced by softer rail pad stiffness. On the other hand, because the resonance frequency due to rail pad stiffness becomes lower by the stiffness change, the decrease of rail vibration decay rate along the rail caused an increase in noise above the $800 \mathrm{~Hz}$ band.

When these three parameters were changed together, the sound pressure level changed in the frequency ranges where these parameters affected noise levels. There was a noise reduction of approximately $10 \mathrm{~dB}$ around the $250 \mathrm{~Hz}$ band. 
These results suggest that it is possible to reduce impact noise from rail joints with appropriate adjustment of track parameters. However, because noise can increase at some frequencies, careful investigation is required when modifications are applied.

\section{Conclusions}

In order to investigate the impact noise characteristics around rail joints, the noise and vibration related to the impact noise during train passage were measured in the section with the joint for evaluation. Also, estimations were made of the influence of track parameters on impact noise using a prediction model. The following conclusions were obtained:

(a) In the section with the joint, which formed the object of this study, when one of the two rails was excited, the rails vibrated independently at frequencies above $400 \mathrm{~Hz}$, although they were both fastened together by a fishplate.

(b) The sleepers in the same section containing a joint vibrated with different amplitudes.

(c) The impact noise and rail vibrations were insensitive to difference in car weight. The power of the impact noise was proportional to train velocity to the power of 2.4 .

(d) In terms of contribution to noise, sleepers formed the major source of noise below the $500 \mathrm{~Hz}$ band, while rail noise dominated above the $1 \mathrm{kHz}$ band.

\section{Authors}

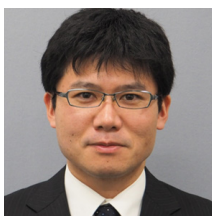

Takeshi SUEKI, Dr. Eng.

Senior Researcher, Noise Analysis Laboratory, Environmental Engineering Division

Research Areas: Railway Noise

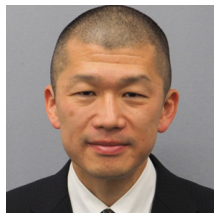

Toshiki KITAGAWA, Ph. D.

Senior Chief Researcher, Laboratory Head, Noise Analysis Laboratory, Environmental Engineering Division Research Areas: Railway Noise (e) Impact noise can be reduced by modifying track parameters.

\section{Acknowledgment}

The authors would like to express their sincere gratitude to the East Japan Railway Company for their support in the field tests.

\section{References}

[1] Kitagawa, T., Murata, K., Kawaguchi, T., Tanaka, S. and Nagakura, K., "Experimental and theoretical studies on impact noise generation due to rail joints," Proceedings of the 11th International Workshop on Railway Noise, pp. 53-60, 2013.

[2] Murata, K., Nagakura, K., Kitagawa, T., Tanaka, S., Kawaguchi, T., "An investigation of impact noise due to rail joints," Proceedings of J-RAIL2012, pp. 325-328, 2012 (in Japanese).

[3] D. J. Thompson, Railway noise and vibration: Mechanisms, modeling and means of control, Elsevier Ltd., 2009

[4] T. X. Wu, and D. J. Thompson, "On the impact noise generation due to a wheel passing over rail joints," Journal of Sound and Vibration, Vol.267, No.3, 2003, pp. $485-496$.

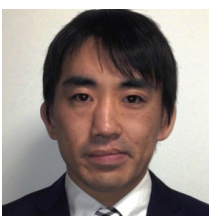

Tsugutoshi KAWAGUCHI

Assistant Senior Researcher, Noise Analysis Laboratory, Environmental Engineering Division Research Areas: Railway Noise 